International Journal of Advanced Biological and Biomedical Research Available online at http:www.ijabbr.com

Volume 8, Issue 2 (2020) pp. 112-127

DOI: 10.33945/SAMI/IJABBR.2020.2.2

Original Article

\title{
The Effect of Halogen Atoms at Propanoate Anion on Thermo Physical, Vibrational Spectroscopy, Chemical Reactivity, Biological Properties of Morpholinium Propionate Ionic Liquid
}

\section{Sunanda Paul1, Ajoy Kumer2,*, Md. Nuruzzaman Sarker3, Mohammad Jahidul Islam ${ }^{3}$}

${ }^{1}$ Department of Biochemistry and Molecular Biology, University of Chittagong, Chittagong,Hathazari-4334, Bangladesh

${ }^{2}$ Department of Chemistry, European University of Bangladesh, Dhaka-1216, Bangladesh

${ }^{3}$ Department of Physics, European University of Bangladesh, Dhaka-1216, Bangladesh

*Corresponding Author E-mail: kumarajoy.cu@gmail.com

Received: 25 June 2019, Revised: 20 August 2019, Accepted: 17 September 2019

\begin{abstract}
The morpholinium cation based ionic liquids are designed to evaluate the thermophysical, chemical reactivity, and biological activity. To estimate and design the bioactive ILs, propionate and trihalopropanoate were considered under theoretical study by Density Functional Theory (DFT). To make effect of halogens atom on anion, propionate, trifluro propionate, tribromo propionate, and triiodo propionate, were to take for optimization. Some thermodynamic and thermophysical properties such as free energy, entropy, dipole moment, binding energy, nuclear energy, electronics energy, and heat of formation were calculated using DFT method and make a comparative effect for halogen atoms activity on anion. Quantitative Structure Activity Relationship (QSAR) like charge density, surface area grid, volume, LogP, polarizability, refractivity, and molecular mass were simulated and recorded, from which the biological activity was calculated. The chemical reactivity like HOMO, LUMO, HUMO-LUMO gap, ionization potential, hardness, softness electronegativity and electron affinity were calculated. The vibrational spectroscopy and UV spectroscopy data provide them the identification and characterization.
\end{abstract}

Key words: Morpholine, DFT, HOMO, LUMO, QSAR, Vibrational spectroscopy

\section{Introduction}

The main focusing point of morpholine is on rubber and color industries use as additive materials (Martini and Whistler, 2007) which is used as a corrosion inhibitor, additive and used to synthesize other chemicals. Morpholine is colorless and free of suspended matter (Grotenbreg et al., 2004; Lanman and Myers, 2004). Due to its low expensive and polarity, its usage is speeded as a solvent in organic synthesis, reactions and chemical industry researches. It is also used in the manufacture of paper, glass, soap, detergent, 
dye and synthetic fiber. The second key point of morpholine is a heterocyclic chemical compound containing both of nitrogen and oxygen atoms, which indicates amine and ether functional groups. Morpholine is a base due to the presence of the amine showing nucleophilic character typical of secondary amines (Wang and Tabor, 1988; Freitas et al., 2014).

Recently, biodegradation and isolation of pure cultures of degrading bacteria of morpholine has been established. As the nitrogen containing heterocyclic has drawn considerable attention of the researchers in the past few decades owing to their high therapeutic values, the key basis point of morpholine is as nitrogen and oxygen containing heterocyclic compound have a pharmaceutical potential drug and therapy. Whether owing to their interesting biological properties they are very often involved as key components in biological processes. On the other hand, Ionic Liquids are considered as green and sustainable molecules with low toxicity containing the carboxylate anions (Hossain et al., 2015; Hossain and Kumer, 2017; Hossain and Kumer, 2017; Hossain, 2017; Hossain, 2018) which have also bioactivity against different human pathogenic bacteria and fungi. Morpholine is converted in the morpholinium cation with different organic anion to form morpholinium ionic liquids and were used as bioactive molecules (Izquierdo, 1979). Another main fact due to the vital role as a pharmaceutical drug and huge bad impact on the human body, the new of morpholinium based Ionic Liquids as a new target of scientists for drug discovery due to tunable thermo-physical and chemical properties (Newington et al., 2007).

All living organisms are found and lived in the open system and their biological process is to relate with irreversible thermodynamic process where both the change of energy and mass take place in surrounding. The thermodynamic has influence the final and initial state of transformation in any biological process that is why some thermodynamical and physiochemical properties have to outcome on biological activity.

Recently, some physical and thermophysical, reaction mechanism and chemical kinetics research were been being in views of DFT which is enough to predict theoretical study and design the new bioactive molecules with their biological properties that is why in this work, is focused on concepts and theoretical study on thermophysical, thermochemical, chemical reactivity, and biological activity. In our previous work, using DFT method, the chemical reactivity, thermophysical HOMO, LUMO, HOMO- LUMO gap, and biological activity were calculated for morpholinium nitrate, nitrite, formate, and acetate ionic liquid crystals, Cannabinoids (CBD) and Tetrahhdrocannabinol (THC), tributylmethylphosphonium cation based anticancer active tri(pentafluroethyl)flurophosphate, tetrafluoroborat, tetrafluorphosphate and chloride to obtain phosphonium ionic liquid crystals, and some aromatic carboxylic acids (Kumer et al., 2019; Kumer et al., 2019; Kumer et al., 2019; Islam, 2019; Kumer et al., 2019; Islam et al., 2019). In this study, taking into account all the benefits of designing new drug molecule, to save time and cost, the computational chemistry is used to determine the thermophysical, chemical and biological activity of some morpholinium propanoate and halopropanoate ILs. To doing this work, morpholinium propanoate and halopropanoate ILs were optimized with DFT/B3LYP. The physiochemical properties, HOMO, LUMO, HOMO-LUMO gap and LogP play the role of the chemical reactivity, biological activity (Kumer et al. 2017; Kumer, 2019; Kumar, 2019). The LogP predicts the hydrophilicity or hydrophobicity of molecule which is considered as the parameters of toxicity. The 
HOMO and LUMO energy level of the complex was calculated at B3LYP of DFT. On the other hand, the QSAR study provides information on biological and pharmacokinetics by which these molecules are considered as a new drug or bioactive molecule.

\section{Materials and methods}

\section{Simulation method}

In case of drawing molecule, drawing default was selected, builds and analyzed different molecular structures and determined the molecular, electronic, and biological properties. In order to create the spatial chemical structure of each calculated molecule, the two-dimensional structure of the molecule shall be built step-by-step by drawing. Then hydrogen atoms are automatically added from building option. The first step in getting the main characteristic parameters of molecules is to optimize the molecular structure to obtain a configuration characterized by minimum energy.

In sitting the DFT was fixed via 6G-31G*, and B3-LYP (Howard et al., 1994). For this calculation, model build was done at first, then fixed the 6G-31G*, total charge zero, spin multiplicity one, UHF, convergence limit is 1e-006. The cut off is fixed 1e-006 with core Hamialtaian calculation and done geometry optimization to record the data of free energy, entropy, dipole moment, binding energy, nuclear energy, electronics energy, the heat of formation, the HOMO, LUMO, 3D mapped structure of electrostatic potential. The QSAR properties were calculated.

Using the computing in vibrational optimization, the UV visible spectroscopy and IR spectroscopy were optimized and recorded as peak.

For estimating a comparative study for halogen atom effect, a statistical analysis were done using excel file in case of thermophysical, chemical reactivity, and biological activity.

\section{Result and discussion}

\section{Optimized structure}

The symmetry is a very powerful tool established on the basis of molecular symmetry. The Molecule belongs to the class asymmetry, the molecules of this group are nonplanar and they have more than one element of symmetry and the plane of the molecule. In Figure 1, the IL01, IL0, IL03, and IL04 are presented of a molecular orbital diagram having both of molecular symmetry and asymmetry properties.

\section{Thermophysical properties}

The binding free energy of the optimized molecules is calculated by performing docking process. The molecule with minimum binding energy will have the maximum binding affinity. The binding free energy of the designed molecules is obtained by eliminating the energy of the main molecule. Having the maximum binding affinity, indicating as the best molecule for drug leads molecules targeting computationally. We can find out the drug binding affinity by using the fitness of the drug, which can bind to the target molecule during the docking process and the second way is using Gibbs free energy calculations. According to this more negative value, we can consider a more effective drug.

$$
\text { Morpholinium propionate (IL01) Morpholinium-3,3,3-trifluropropionate (IL02) }
$$




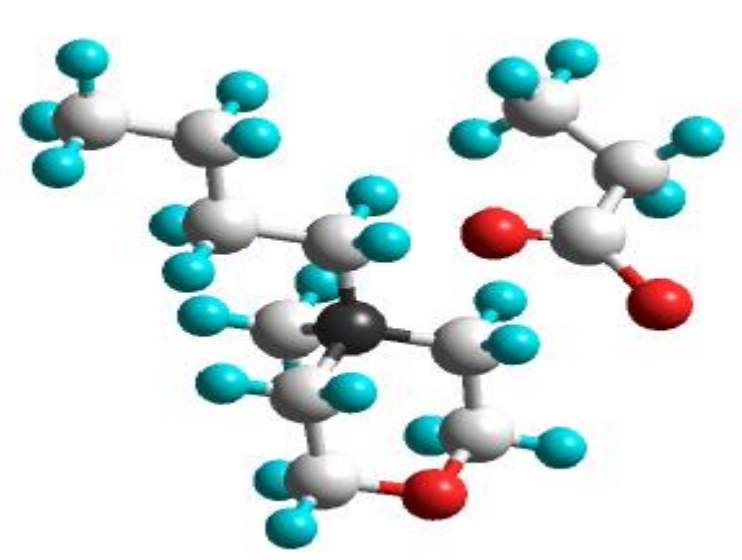

Morpholinium -3,3,3-trichloropropionate (IL03)

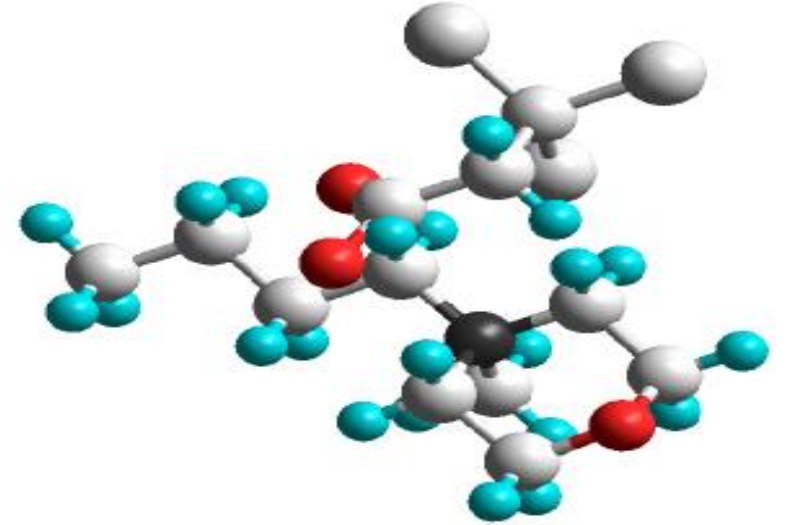

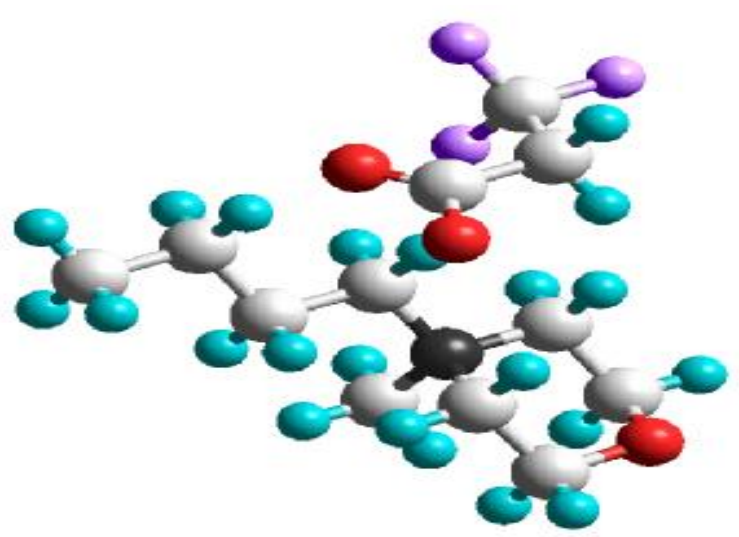

Morpholinium -3,3,3-tribromopropionate (IL04)

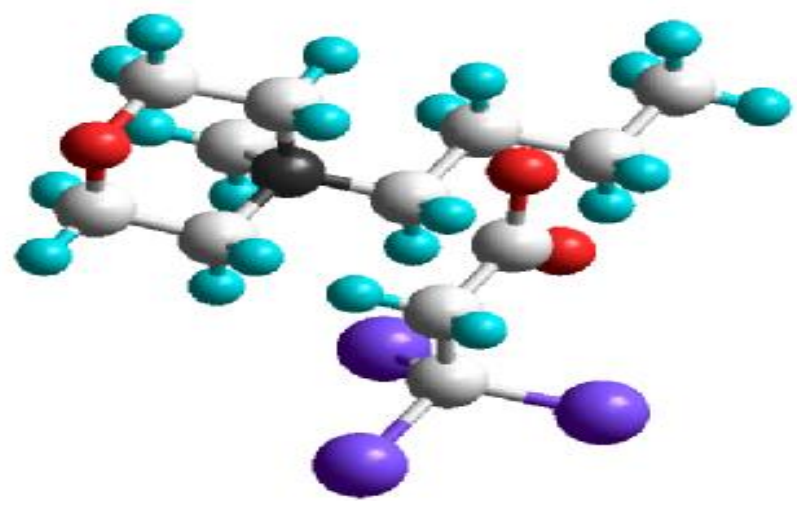

Figure 1. Optimized structure in a cylinder shape, Color: Red is oxygen, cyan is hydrogen, carbon is brown, chlorine is big white ball, fluorine is small shape and light violet, bromine is violet and nitrogen is black

The bond dipole moment is the idea of an electric dipole moment to measure the polarity of a chemical bond within a molecule. In view of pharmaceutical industries, the dipole moment was a parameter for the drug by which a drug is to be used open or close packet. If any drug has a dipole moment, it can be able to absorb the ultraviolet light and have a possibility of properties. The four optimized ILs show zero dipole moment so that it can be used without any protection from UV or sunlight. The all data is given Table 1 and Table 2 .

\section{Temperature effect on entropy and heat of capacity}

The entropy and heat of capacity are the important parameters for physiochemical properties. The entropy indicates the disturbance of molecular system. From the Table 2 , the entropy was found at $273 \mathrm{~K}$ where chloro propanoate shows the highest and gradually increases with temperature. The second is fluro propanoate, then propanoate, and bromo propanoate. The heat of capacity is similar to entropy.

Table 1. Thermophysical properties 


\begin{tabular}{ccccc}
\hline \hline Properties & IL01 & IL02 & IL03 & IL04 \\
\hline $\begin{array}{c}\text { Total energy, } \\
\text { (kcal/mol) }\end{array}$ & -65750.8014 & -95157.4447 & -86584.3982 & -93783.2530 \\
Free energy, & -65750.8014 & -95157.4447 & -86584.3982 & -93783.2530 \\
$\quad(\mathrm{kcal} / \mathrm{mol})$ \\
$\begin{array}{c}\text { Dipole moment, (D) } \\
\text { RMS gradient, } \\
\quad(\mathrm{kcal} / \mathrm{mol})\end{array}$ & 0 & 0 & 0 & 0 \\
$\begin{array}{c}\text { Binding energy, } \\
\quad(\mathrm{kcal} / \mathrm{mol})\end{array}$ & -3780.7146 & -3823.0397 & -3712.0252 & -3657.3136 \\
$\begin{array}{c}\text { Heat of formation, } \\
\quad(\mathrm{kcal} / \mathrm{mol})\end{array}$ & -135.8076 & -277.7687 & -137.0252 & -88.4926 \\
$\begin{array}{c}\text { Electronic energy, } \\
(\mathrm{kcal} / \mathrm{mol})\end{array}$ & -465822.8798 & -631445.8035 & -614221.6903 & -603145.3078 \\
$\begin{array}{c}\text { Nuclear energy, } \\
(\mathrm{kcal} / \mathrm{mol})\end{array}$ & 400072.0694 & 536288.3587 & 527637.292 & 509362.0547 \\
\hline
\end{tabular}

Table 2. Entropy and Heat capacity in different temperature

\begin{tabular}{ccccccc}
\hline Entropy & $\begin{array}{c}\text { 273 K } \\
\text { Heat capacity, } \\
\text { (kcal/mol-deg) }\end{array}$ & Entropy & $\begin{array}{c}\text { 298 K } \\
\text { Heat capacity, } \\
\text { (kcal/mol-deg) }\end{array}$ & Entropy & $\begin{array}{c}\text { Heat } \\
\text { capacity, } \\
\text { (kcal/ mol- } \\
\text { deg)) }\end{array}$ \\
\hline IL01 & 0.1240 & 0.05820 & 0.1295 & 0.0637 & 0.1351 & 0.0693 \\
IL02 & 0.1359 & 0.0657 & 0.1421 & 0.0714 & 0.1482 & 0.0772 \\
IL03 & 0.1506 & 0.07228 & 0.1574 & 0.0779 & 0.1640 & 0.0836 \\
IL04 & 0.0820 & 0.0128 & 0.0833 & 0.0140 & 0.0846 & 0.0151 \\
\hline
\end{tabular}

\section{Chemical reactivity by HOMO, LUMO}

The energy levels of the molecular orbital order HOMO and LUMO give information on the possible electronic transition, and also indicated the electrophilic and nucleophilic attraction region in the molecule highlighted in Figure 2 by green color and blue color and Table 3. The LUMO-HOMO gap is the most important parameter for determining chemical reactivity. The shorter LUMO- HOMO gap is considered as the high reactivity.

Table 3. Data of HOMO, LUMO in different energy level

\begin{tabular}{ccccc}
\hline & IL01 & IL02 & IL03 & IL04 \\
\hline HOMO (0), eV & -8.7441 & -8.2262 & -8.1045 & -7.6583 \\
HOMO (-1), eV & +0.1110 & 0.3245 & -0.0160 & -0.4918 \\
HOMO (-2), eV & +1.4627 & 0.9469 & 0.4243 & 0.0310 \\
HOMO (+1), eV & N/A & N/A & N/A & N/A \\
HOMO (+2), eV & N/A & N/A & N/A & N/A \\
LUMO, (0) eV & 0.1110 & 0.3245 & -0.0160 & -0.4918 \\
LUMO, (-1) eV & -8.7441 & -8.2262 & -8.1045 & -7.6583 \\
LUMO, $(-2) \mathrm{eV}$ & -9.0227 & -105965 & -10.3556 & -10.27784 \\
\hline
\end{tabular}


In addition, according to Koopmans' theorem the energy gap, Egap, defined as the difference between HOMO and LUMO energy (koopmans, 1934). It magnitude is recorded in Table 4.

$$
E_{g a p}=\left(E_{\text {LUMO }}-E_{\text {HOMO }}\right) \approx I P-E A
$$

Table 4. Data of HOMO, LUMO gap, eV in zero nergy level

\begin{tabular}{ccccc}
\hline & IL01 & IL02 & IL03 & IL04 \\
HOMO, (0), eV & -8.7441 & -8.2262 & -8.1045 & -7.6583 \\
LUMO, (0), eV & 0.1110 & 0.3245 & -0.0160 & -0.4918 \\
HOMO, LUMO gap, eV & 8.8551 & 8.2262 & 8.0885 & 7.1665 \\
\hline
\end{tabular}

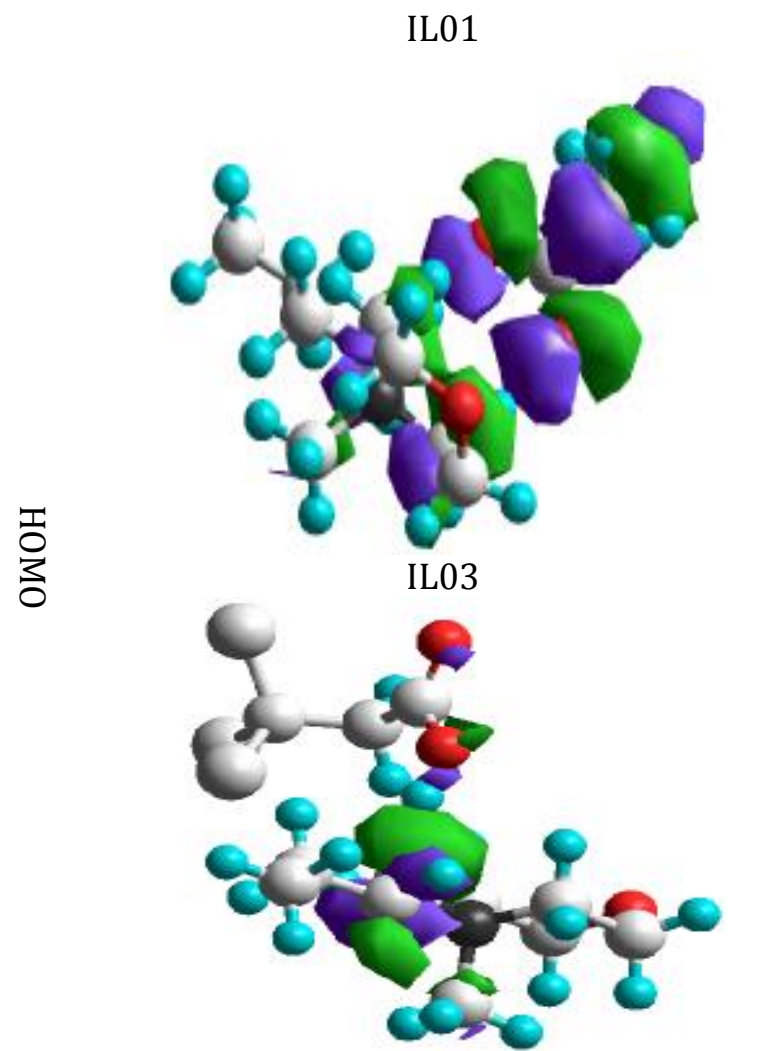

IL01

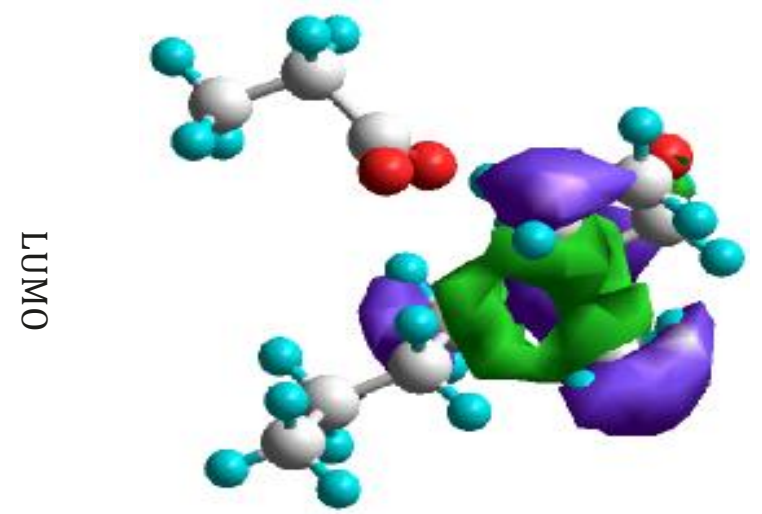

IL03
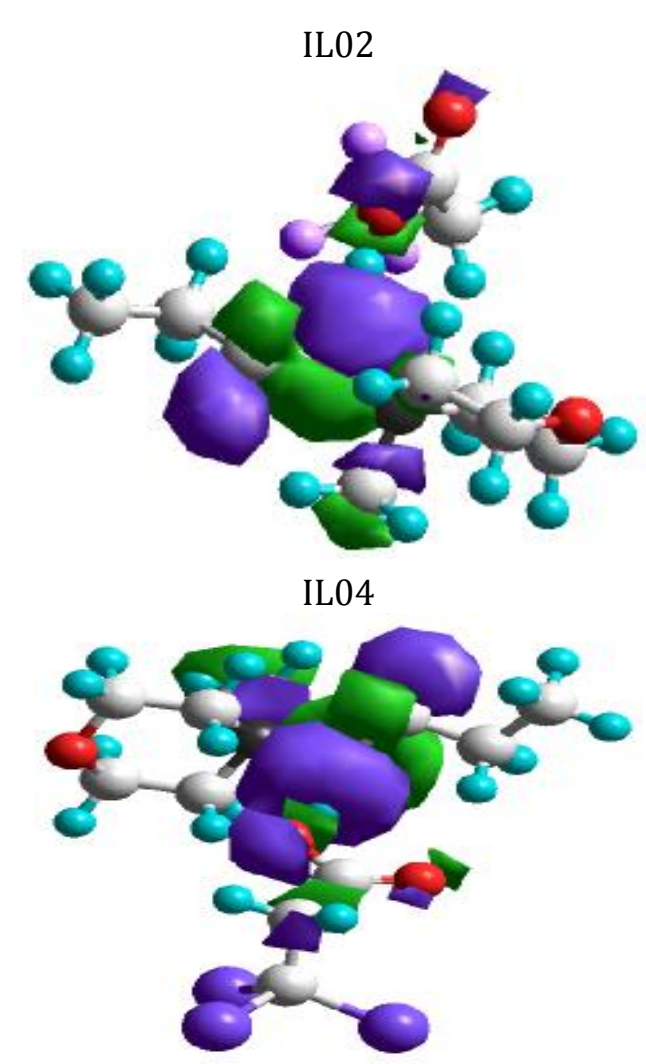

IL02

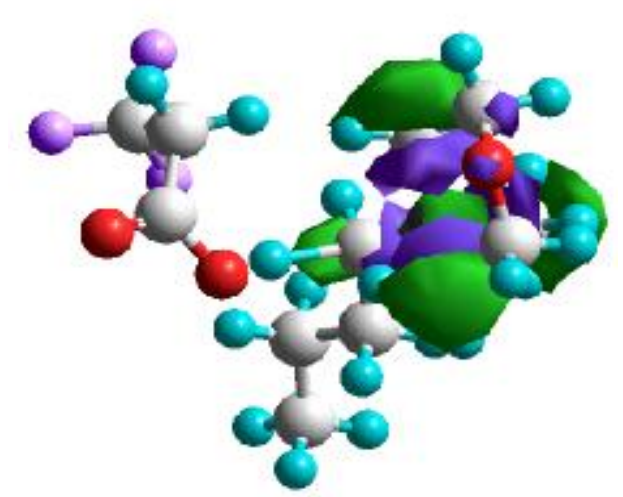

IL04 

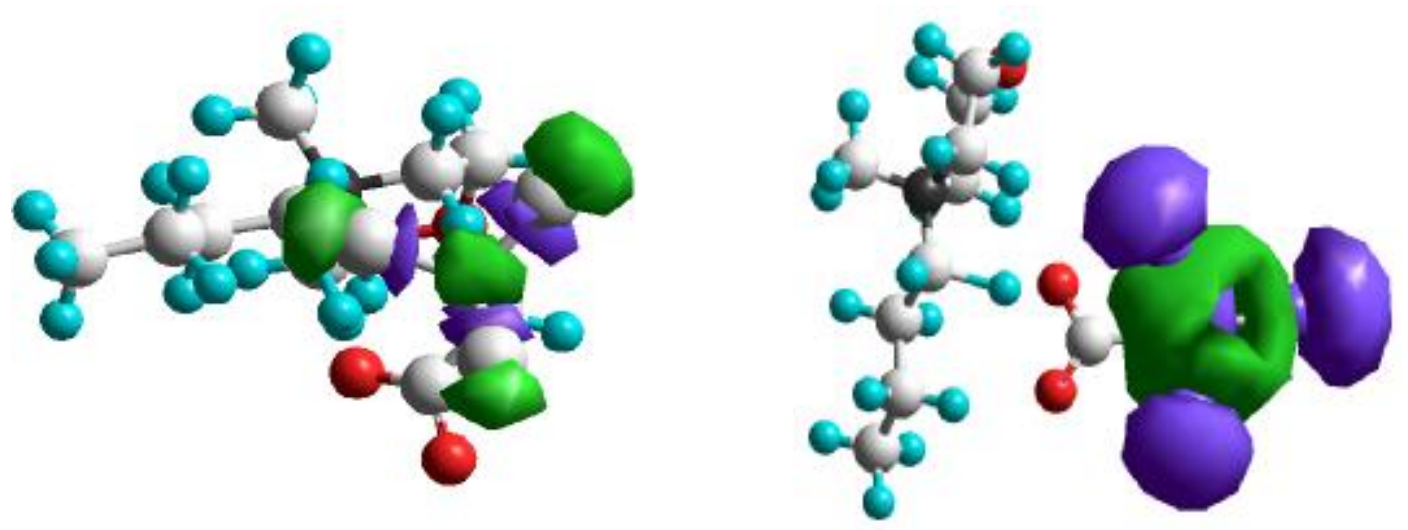

Figure 2. HOMO, LUMO picture

\section{The distribution electrostatic potential energy and orbital structure}

The stability of the studied molecular structure is given by the higher negative values of total energy. The biological activity of a compound can be estimated on the basis of the energy difference $\Delta \mathrm{E}$ frontier orbitals. This difference, $\Delta \mathrm{E}$ represents the electronic excitation energy which is possible in a molecule. According to the mechanism of antimicrobial activity and antimicrobial agents of bioactive molecules, the positive charge end of molecules is responsible to damage the plasma membrane of pathogens (Timofeeva and Kleshcheva, 2011). To kill pathogens, the region of molecules was used in the positive charge area of the molecule. In this case, the most important factors are explained that the higher surface area having a positive charge is considered as the high antimicrobial activity. The electrostatic potential in view of 3D mapped structure indicates positive and negative charge region and charged surface area in a molecule(Collins, 1997) that is considered as the best tools to estimate the biological activity parameters (Stürzebecher et al., 1999). The surface distribution of molecular electrostatic potential is an indicator of the specific reactive regions of the molecule given in Figure 3 and Table 5.

IL01

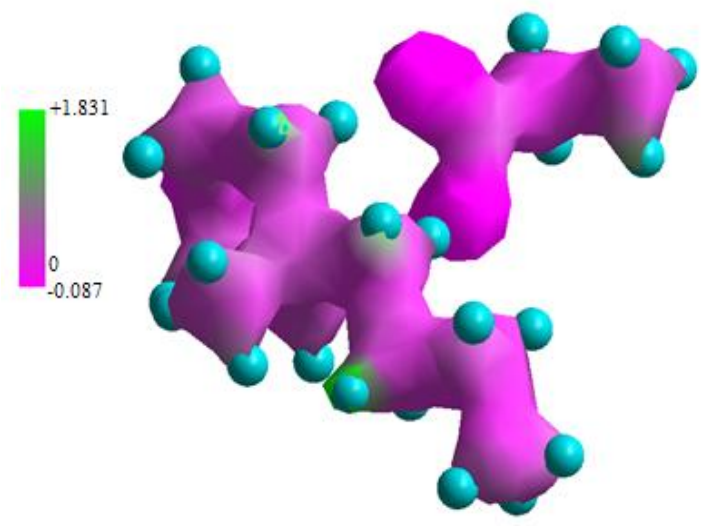

IL03

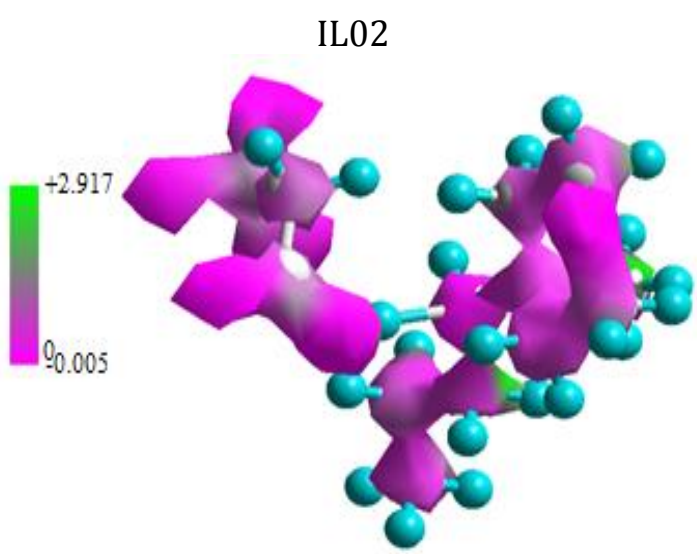

IL04 


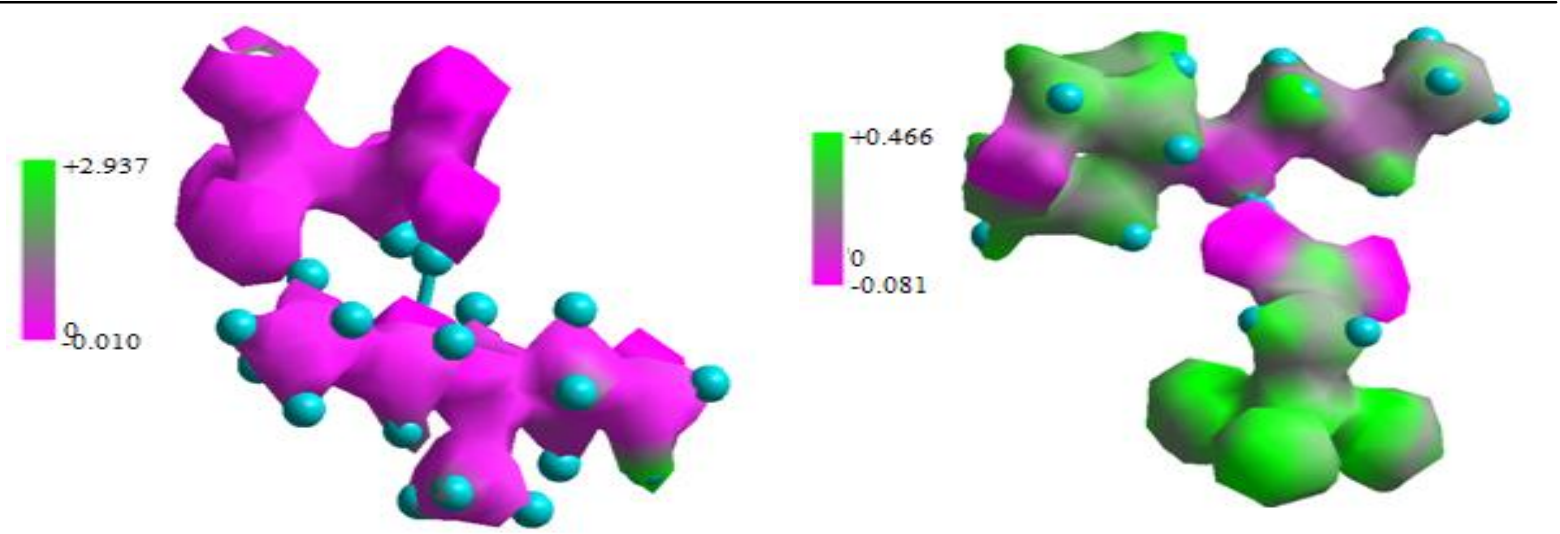

Figure 3. Electrostatic Potential 3D mapped picture

Table 5. Data of electrostatic potential energy difference of two levels

\begin{tabular}{ccccc}
\hline & IL01 & IL02 & IL03 & IL04 \\
\hline E1 & +1.831 & +2.917 & +2.937 & +0.466 \\
E2 & -0.087 & -0.005 & -0.010 & -0.081 \\
$\Delta \mathrm{E}=$ E2-E1 & -1.918 & -2.922 & -2.947 & -0.547 \\
\hline
\end{tabular}

\section{Vibrational spectrum}

The Vibrational spectra of morpholinium based ILs confirmed the presence of carboxylate (COO-) from the symmetric and asymmetric stretching peak about $~ 1770$ $\mathrm{cm}^{-1}$ to $\sim 1550 \mathrm{~cm}^{-1}$ respectively, with the former being overlapped by $\mathrm{N}-\mathrm{H}$ vibrations. The broad absorption around $\sim 3335-3500 \mathrm{~cm}^{-1}$ can be assigned the presence of $\mathrm{N}-\mathrm{H}$ vibrations in the morpholinium ion and shown in Figure 4, Table 6.

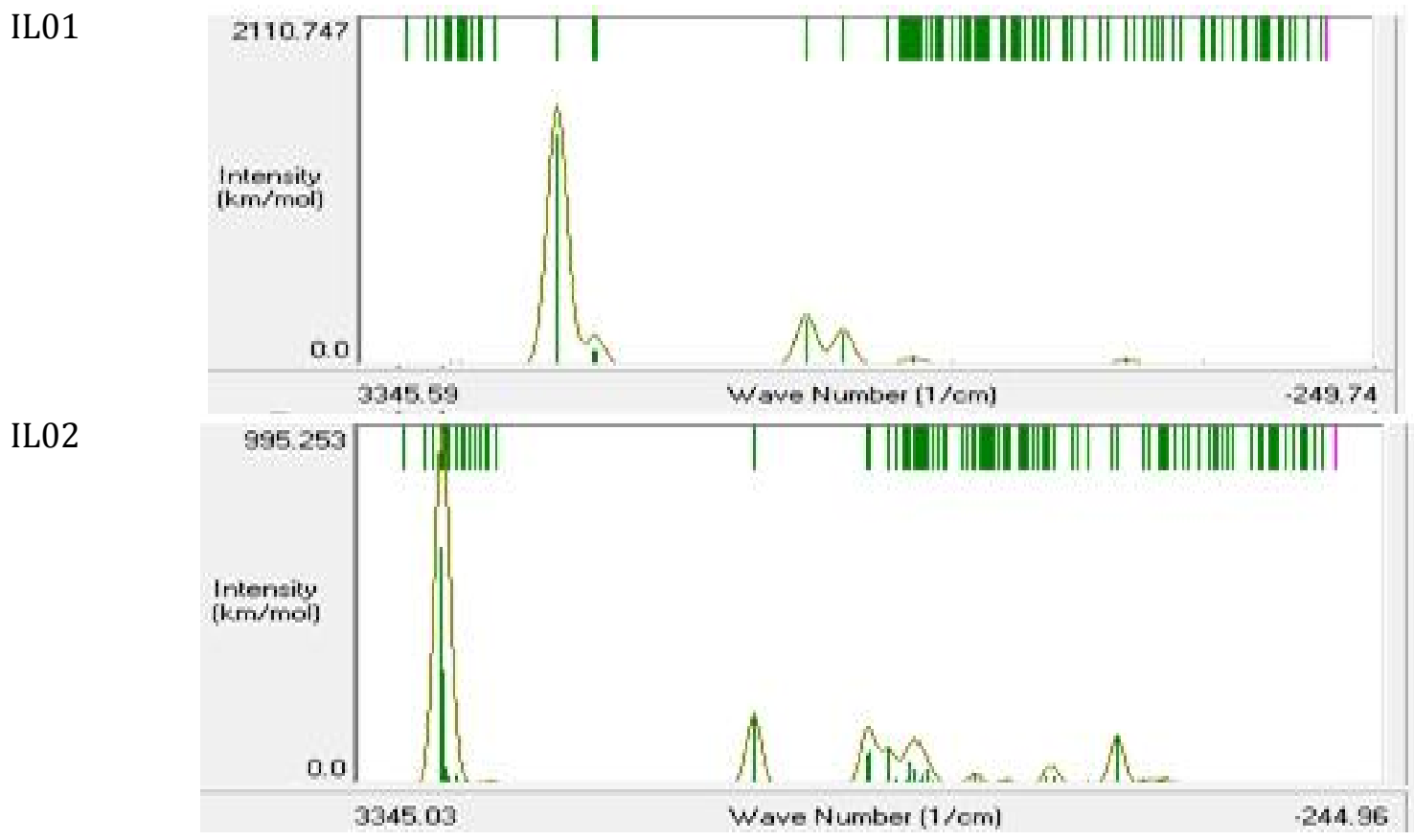




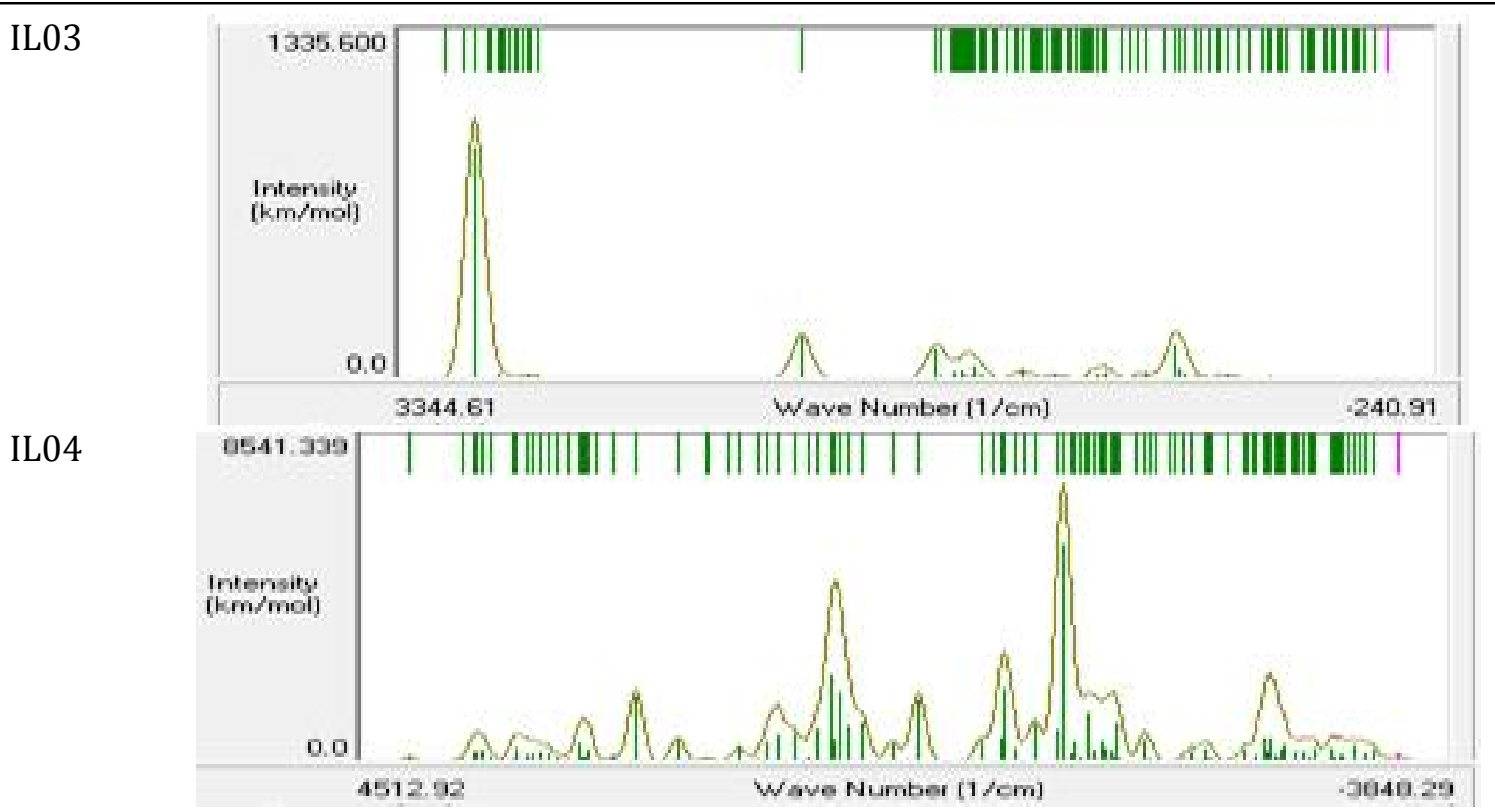

Figure 4. Vibrational spectrum of morpholinium based ILs

Table 6. Data for a vibrational spectrum of morpholinium based ILs

\begin{tabular}{cccccc}
\hline & Normal mode & Degeneracy & Frequency & Intensity & Symmetry \\
\hline IL01 & 1 & 1 & -86.32 & 0.006 & 1 A \\
IL02 & 1 & 1 & -81.78 & 0.007 & 1 A \\
IL03 & 1 & 1 & -77.93 & 0.014 & 1 A \\
IL04 & 1 & 1 & -3468.23 & $\mathbf{1 8 4 . 2 1 4}$ & 1 A \\
\hline
\end{tabular}

\section{UV-visible Spectrum}

UV-visible Spectrum provides a powerful technique by which the nature of nitrogen ion with anion bonding may be identified. A remarkable covalency between almost all of the upper filled molecular orbitals of the ligand cluster orbitals of suitable symmetry can be calculated (Canadell, 1990). The UV-visible spectrum of the morpholinium based ILs shows a strong transition (near 150 and $190 \mathrm{~nm}$ ) shown in Figure 5 and different conditions in Table 7.

IL01

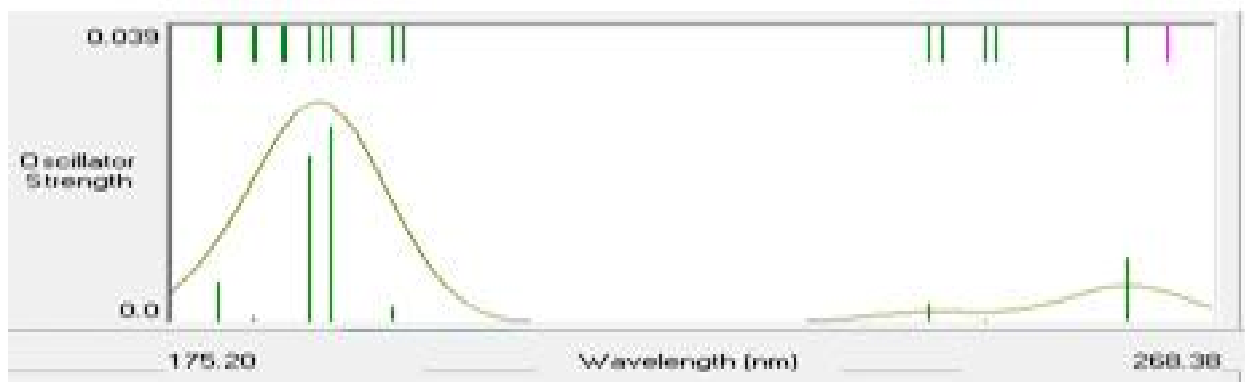




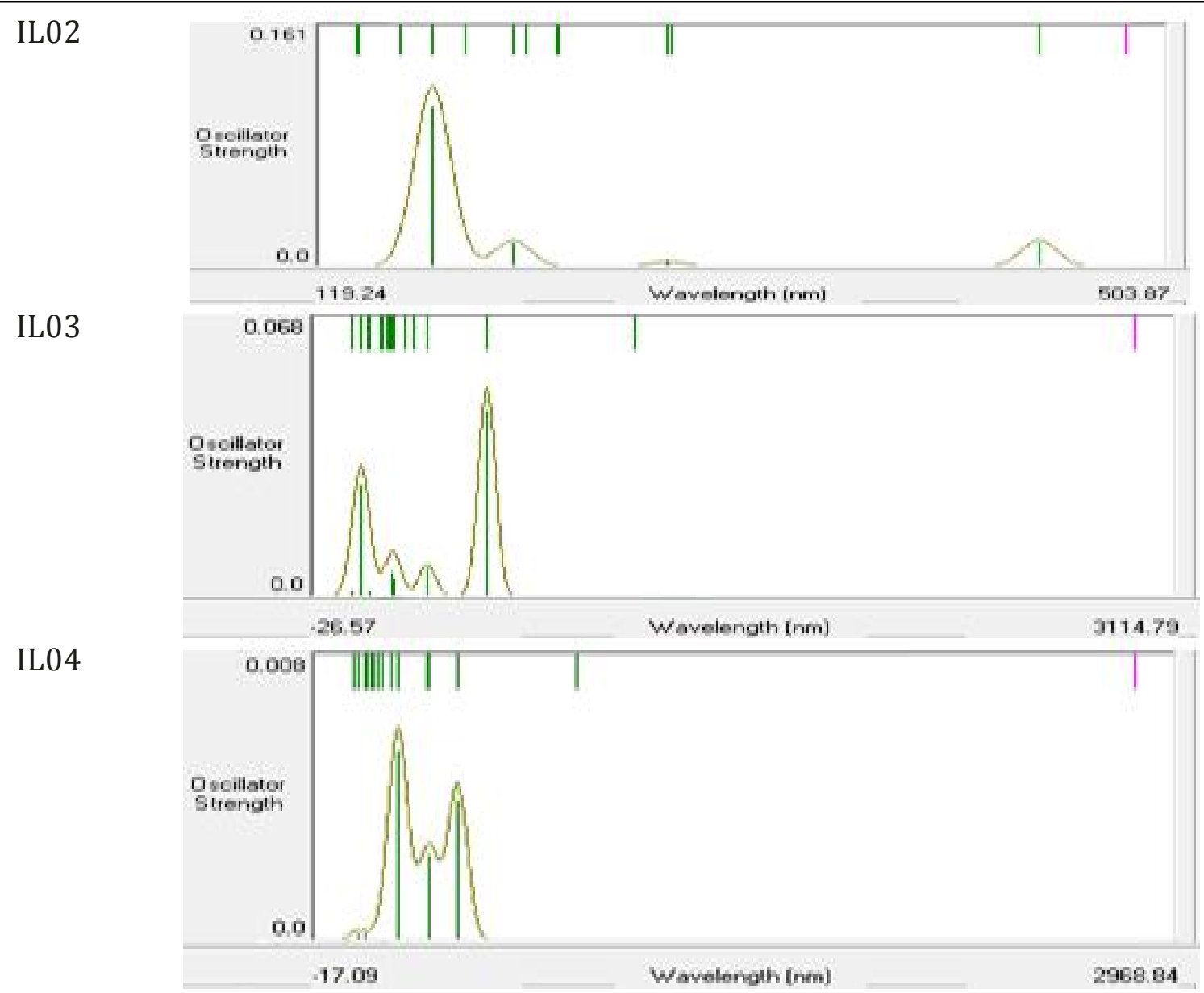

Figure 5. UV-visible Spectrum

Table 7. Data for different transition state, spin multiplicity, wavelength and oscillator strength for UV -visible spectrum

\begin{tabular}{cccccc}
\hline & Transition & Degeneracy & Spin Multiplicity & Wavelength & Oscillator Strength \\
\hline IL01 & 1 & 1 & Triplet & 264.14 & 0.0 \\
IL02 & 1 & 1 & Triplet & 486.38 & 0.0 \\
IL03 & 1 & 1 & Triplet & 2972.0 & 0.0 \\
IL04 & 1 & 1 & Triplet & 2833.12 & 0.0 \\
\hline
\end{tabular}

\section{Biological activity and QSAR study}

The stability of the studied molecular structure is given by the higher negative values of total energy. The biological activity of a compound can be estimated on the basis of the energy difference $\Delta \mathrm{E}$ frontier orbitals given in Table 5. This difference, $\Delta \mathrm{E}$ represents the electronic excitation energy which is possible in a molecule. The electrostatic potential in view of the 3D mapped structure indicates positive and negative charge region and the charged surface area in a molecule that is considered as the best tools to estimate the biological activity parameter (Stürzebecher et al., 1999). 
According to the mechanism of antimicrobial activity and antimicrobial agents of bioactive molecules, the positive charge end of molecules is responsible to damage the plasma membrane of pathogens (Timofeeva and Kleshcheva, 2011). To kill the different human pathogenic microorganism, the region of molecules was used the positive charge area of the molecule. In this case, the most important factors are explained that the higher surface area having a positive charge is considered as the high antimicrobial activity.

The three-dimensional geometry of molecular electrostatic potential distribution highlights the existence of three regions with increased electronegativity in the whole molecule of IL01, IL02 and highly positivity in IL03 and IL04 shown in table 8 and which play a role in their coupling to different structures in which ions are positively charged.

Table 8. Data for QSAR study

\begin{tabular}{ccccc}
\hline & IL01 & IL02 & IL03 & IL04 \\
\hline Partial charge (e) & 0.00 & 0.0 & 0.0 & 0.0 \\
Surface Area (grid), kcal/mol & 475.68 & 478.47 & 511.76 & 558.65 \\
Volume, $\mathbf{A}^{\mathbf{3}}$ & 783.40 & 791.85 & 871.02 & 954.71 \\
Hydration Energy kcal/mol & 10.99 & -33166180 & -19470140 & -293892400 \\
Log P & 1.52 & 1.77 & 3.40 & 3.59 \\
Refractivity $\mathbf{A}^{\mathbf{3}} \mathrm{kcal} / \mathrm{mol}$ & 61.83 & 61.76 & 75.99 & 84.94 \\
Polarizibility, $\mathbf{A}^{\mathbf{3}} \mathrm{kcal} / \mathrm{mol}$ & 24.47 & 24.20 & 30.26 & 32.35 \\
Mass $(\mathrm{amu})$ & 231.34 & 285.31 & 334.67 & 468.02 \\
\hline
\end{tabular}

\section{Chemical Reactivity}

The HOMO and LUMO energies are used for the determination of global reactivity descriptors. It is important that Ionization potential (I), Electron affinity (A), Electrophilicity ( $\omega)$, Chemical potential $(\mu)$, Electronegativity $(\chi)$, Hardness $(\eta)$ and Softness $(S)$ be put into a Molecular Orbital's framework. We focus on the HOMO and LUMO energies in order to determine the interesting molecular/atomic properties and chemical quantities. In simple molecular orbital theory approaches, the HOMO energy is related to the ionization potential (I) and the LUMO energy has been used to estimate the electron affinity (A) respectively by the following relations and Table 9.

$$
\begin{aligned}
& I=-E_{\text {HoMo }} \\
& A=-E_{\text {LUMO }} \\
& (\mu)=\mathrm{I}+\mathrm{A} / 2 \\
& (\eta)=\mathrm{I}-\mathrm{A} / 2 \\
& (S)=\frac{1}{\eta} \\
& (\chi)=\mathrm{I}+\mathrm{A} / 2 \\
& (\omega)=\frac{\mu^{2}}{2 \eta}
\end{aligned}
$$


Table 9. Chemical reactivity

\begin{tabular}{ccccc}
\hline & IL01 & IL02 & IL03 & IL04 \\
\hline Ionization potential (I), eV & 8.7441 & 8.2262 & 8.1045 & 7.6583 \\
Electron affinity, (A), eV & -0.1110 & -0.3245 & 0.0160 & 0.4918 \\
Chemical Hardness, Ev & 4.4275 & 4.2753 & 4.0442 & 3.5832 \\
Chemical Softness, eV & 0.2258 & 0.2339 & 0.2472 & 0.2797 \\
Electronegativity, eV & 4.3165 & 3.9508 & 4.0602 & 4.0750 \\
Chemical Potential, eV & -4.3165 & -3.9508 & -4.0602 & -4.0750 \\
Electrophilicity Index, eV & 41.258 & 33.3664 & 33.3438 & 29.6847 \\
\hline
\end{tabular}

\section{Statistical analysis and comparison study of halo atom activity}

In case of chemical reactivity, the LUMO-HOMO gap in presence of IL02, IL03 and IL04 are almost same means that with substituting hydrogen atom by chlorine, fluorine and bromine atom in anion, the chemical reactivity is changed poorly. On the other hand, the chemical reactivity of morpholinium propionate is lower than other which means that replacing hydrogen atom with halogens atom has higher reactivity shown in Figure 6.

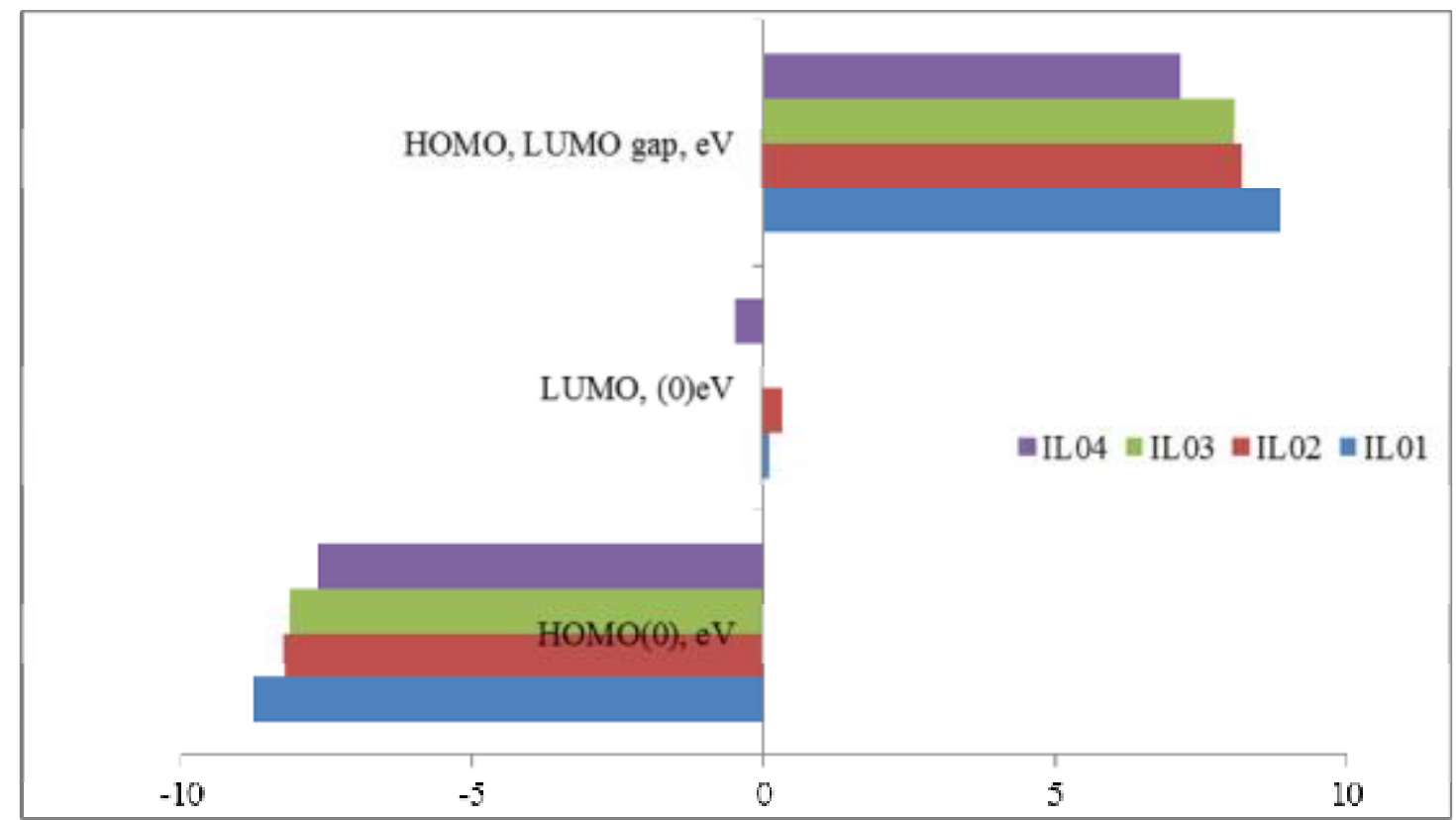

Figure 6. HOMO, LUMO and HOMO LUMO gap

In case of QSAR study, the value of LogP is increased slightly with substituting hydrogen atom by halogen atom that is why they are hydrophobic nature. Also refractivity and polarizibility increased gradually due to substitute hydrogen atom by halogen atom shown in Figure 7.

The most important comparison in thermophysical properties is explained that with replacing hydrogen atom by chlorine, fluorine and bromine atom of propanoate shown in Figure 8. That the total energy, binding energy, free energy, heat of formation, and electronics energy are increasing. 


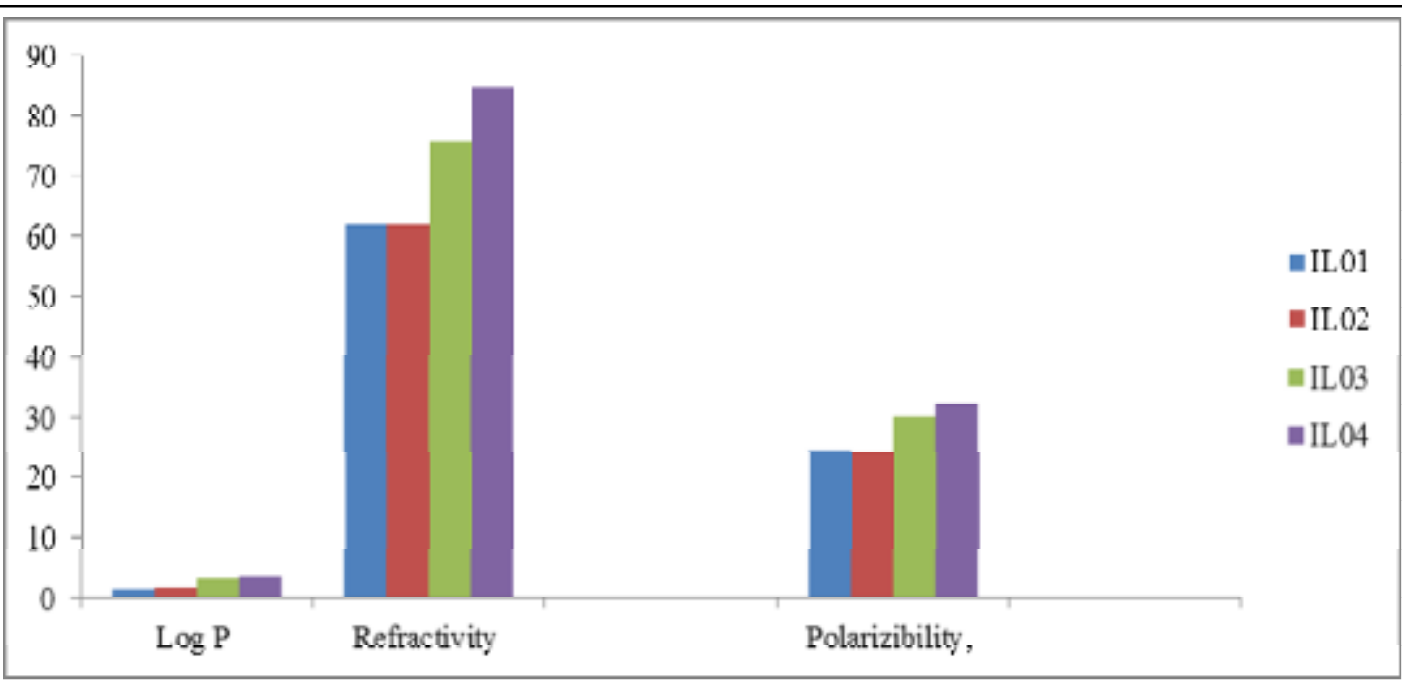

Figure 7. QSAR study

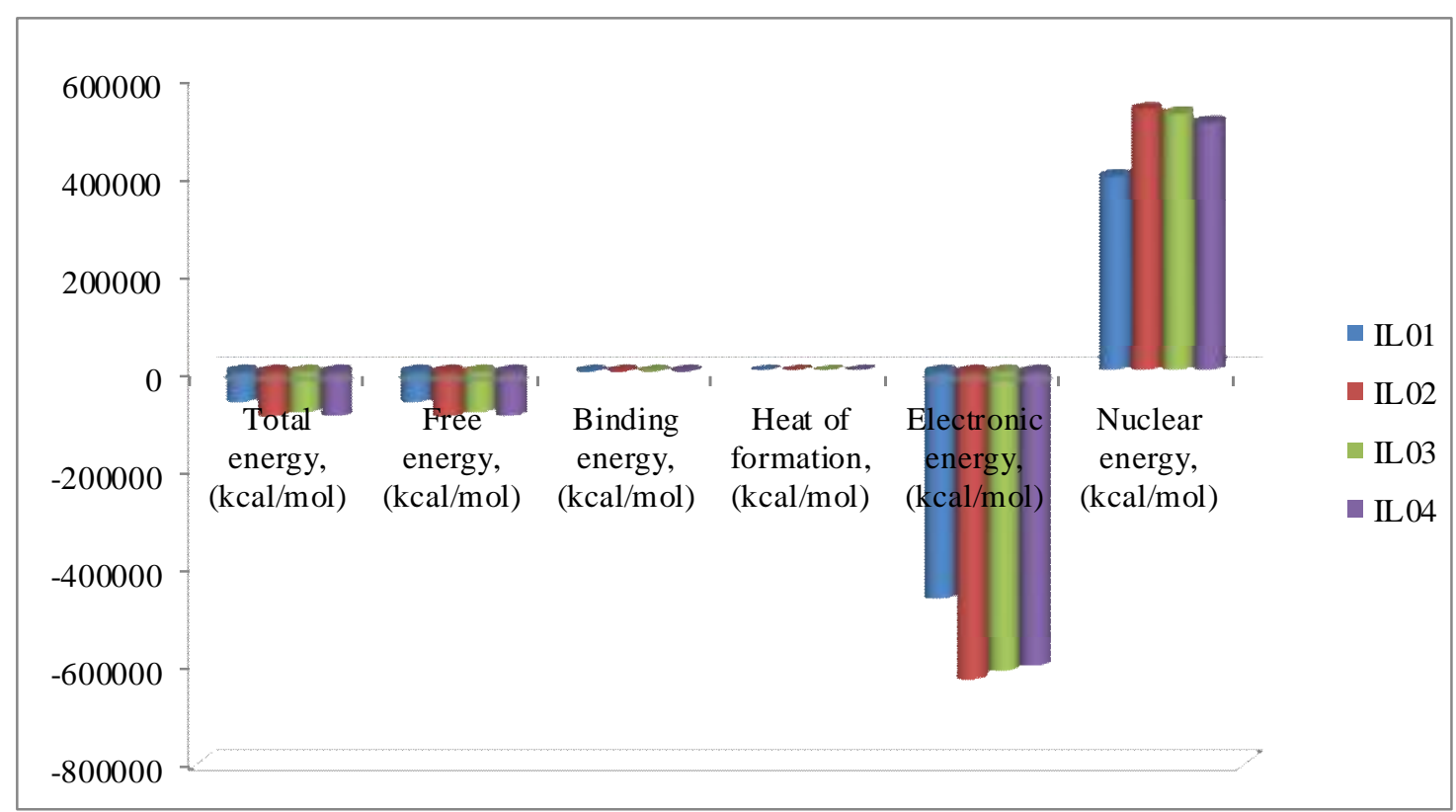

Figure 8. Thermophysical study

In case of chemical reactivity, the ionization potential is declined slightly with adding halogen atoms by replacing hydrogen atom. Also similar trend is observed for electrophilicity index with substituting hydrogen atom by halogen atom shown in Figure 9.

\section{Discussion}

The first point is noted that the thermophysical properties changing in order of IL02 $>$ IL03 $>$ IL04>IL01 means that fluorine atom shows the highest activity then, chlorine, bromine.

The second is the chemical reactivity in terms of HOMO, LUMO, HOMO LUMO gap, electronegativity, hardness, softness, and chemical potential affecting by halo atom on anion. It 
is inverse of physical properties, and reactivity decreases with attaching large halo atom such as IL01>IL02>IL03>IL04.

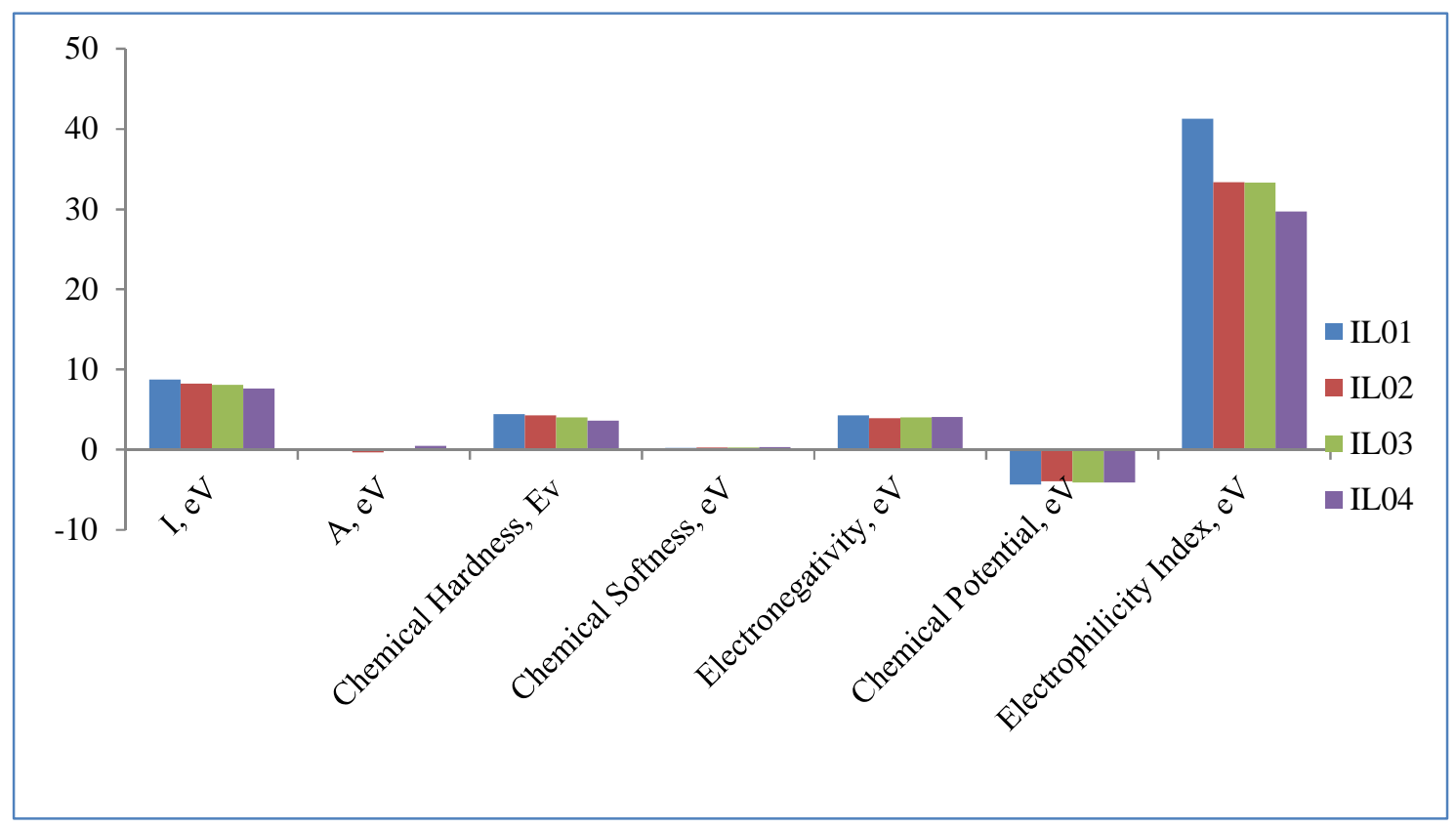

Figure 9. Comparison of chemical kinetics

Finally, the biological activity of morphonium carboxylate Ionic Liquids is to found and their toxicity in term of $\log P$ value is explained. The $\log P$ value is $+1.52,+1.77,+3.40$, and +3.59 for IL01, IL02, IL03, and IL04 respectively which is positive. For this case, all are hydrophobic. The main point is mentioned such way that the hydrophobicity increases with attaching large halo atoms, and order is IL04>IL03>IL02>IL01.

\section{Conclusions}

The most important properties for biological chemistry, reactivity and drug design, the HOMO, LUMO, HOMO-LUMO gap, ionization potential, electron affinity, and electrostatic potential in case of the charge distribution in molecule were optimized and recorded using DFT modeling methods.

It is summarized that the result optimized molecules of morpholinium propionate salts is established as higher chemical reactive and biological active where halo atoms have effect on all physical, thermophysical, chemical and biological activity.

\section{References}

Böhm, M, St rzebecher, J, Klebe, G. (1999). Three-dimensional quantitative structureactivity relationship analyses using comparative molecular field analysis and comparative molecular similarity indices analysis to elucidate selectivity differences of inhibitors binding to trypsin, thrombin, and factor Xa. J. Med. Chem., 42(3):458-477.

Canadell, ER, Ravy, S, Pouget, JP, Brossard, L. (1990). Concerning the band structure of D (M (dmit) 2) 2 (D= TTF, Cs, NMe4); M= Ni, Pd) molecular conductors and superconductors: Role of the M (dmit) 2 Homo and Lumo. Solid State Commun., 75(8):633-638. 
Collins, KD. (1997). Charge density-dependent strength of hydration and biological structure. Biophys. J., 72(1):65-76.

Freitas, VL, Gomes, JRB, Ribeiro da Silva, MDMC. (2014). Energetics and reactivity of morpholine and thiomorpholine: a joint experimental and computational study. J. Chem. Eng. Data, 59(2):312-322.

Grotenbreg, GM, Christina, AE, Buizert, AE, van der Marel, GA, Overkleeft, HS, Overhand, M. (2004). Synthesis and application of carbohydrate-derived morpholine amino acids. J. Org. Chem., 69 (24):8331-8339.

Hossain, MI, Kumer, A. (2017). Synthesis and characterization of ammonium benzilate bioactive ionic liquids and their antimicrobial activity. Asian J. Phys. Chem. Sci., 4(4):1-13.

Hossain, MI, Kumer, A. (2017). Synthesis and Characterization of Ammonium Ionic Liquids and Their Antimicrobial and Computational Overview. Asian J. Phys. Chem. Sci., 3(4):1-10.

Hossain, MI, Kumer, A. (2017). Synthesis and characterization of ammonium benzilate bioactive ionic liquids and their antimicrobial activity. Asian J. Phys. Chem. Sci., 4(4):1-13.

Hossain, MI, Kumer, A. Begum, S. (2018). Synthesis and Characterization of Ammonium Benzoate and Its Derivative Based Ionic Liquids and Their Antimicrobial Studies. Asian J. Phys. Chem. Sci., 5(1):1-9.

Hossain, MI, El-Harbawi, M, Kumer, A. (2015). Acute toxicity of $\mathrm{OH}$ functionalized ionic liquids to the aquatic species. Int. J. Adv. Eng. Sci. Technol., (IJAEST), 4(3):244-249.

Howard, A, J. McIver, et al. (1994). Hyperchem computational chemistry. Hypercube Inc., Waterloo.

Islam, MJ, Kumer, A, Sarker, N, Paul, S, Zannat, A. (2019). The prediction and theoretical study for chemical reactivity, thermophysical and biological activity of morpholinium nitrate and nitrite ionic liquid crystals: A DFT study. Adv. J. Chem. Section A, 2(4):316-326.

Islam, MJ, Sarker, N, Kumer, A, Paul, S. (2019). The Evaluation and Comparison of ThermoPhysical, Chemical and Biological Properties of Palladium(II) Complexes on Binuclear Amine Ligands with Different Anions by DFT Study. Int. J. Adv. Biol. Biomed. Res., 7(4):15334.

Izquierdo, I. (1979). Effect of naloxone and morphine on various forms of memory in the rat: Possible role of endogenous opiate mechanisms in memory consolidation. Psychopharmacology, 66(2):199-203.

Koopmans, YT. (1934). Uber die zuordnung von wellenfunktionen und eigenwerten zu den,einzelnen elektronen eines atoms. Physica, 1(1-6):104-113.

Kumer, A, Ahmed, B, Sharif, A, Al-Mamun, A. (2017). A Theoretical Study of Aniline and Nitrobenzene by Computational Overview. Asian J. phys. Chem. Sci., 4(2):1-12. 
Kumer, A, Sarker, N, Paul, S. (2019). The Simulating Study of HOMO, LUMO, thermo physical and Quantitative Structure of Activity Relationship (QSAR) of Some Anticancer Active Ionic Liquids. Eurasian J. Environ. Res., 3(3):1-10.

Kumer, A, Sarker, N, Paul, S. (2019). The thermo physical, HOMO, LUMO, Vibrational spectroscopy and QSAR study of morphonium formate and acetate Ionic Liquid Salts using computational method. Turkish Computat. Theoret. Chem., 3(2):59-68.

Kumer, A, Sarker, N, Paul, S. (2019). The Theoretical Prediction of Thermophysical properties, HOMO, LUMO, QSAR and Biological Indics of Cannabinoids (CBD) and Tetrahhdrocannabinol (THC) by Computational Chemistry. Adv. J. Chem. Section A, 2(3):190-202.

Kumer, A, Sarker, N, Paul, S. (2019). The theoretical investigation of HOMO, LUMO, thermophysical properties and QSAR study of some aromatic carboxylic acids using HyperChem programming. Int. J. Chem. Technol., 3(1):26-37.

Kumer, A, Sarker, N, Paul, S. Afroza Z. (2019). The Theoretical Prediction of Thermophysical properties, HOMO, LUMO, QSAR and Biological Indics of Cannabinoids (CBD) and Tetrahhdrocannabinol (THC) by Computational Chemistry. Adv. J. Chem., 3(3):1-13.

Kumer, A, Sarker, N, Paul, S. (2019). The theoretical investigation of HOMO, LUMO, thermophysical properties and QSAR study of some aromatic carboxylic acids using HyperChem programming. Int. J. Chem. Technol., 3(1):26-23.

Lanman, BA, Myers, AG. (2004). Efficient, stereoselective synthesis of trans-2, 5disubstituted morpholines. Org. Lett., 6(6):1045-1047.

Martini, L, Whistler, JL. (2007). The role of mu opioid receptor desensitization and endocytosis in morphine tolerance and dependence. Current Opin. Neurobiol., 17(5):556564.

Newington, I, Perez-Arlandis, JM, Welton, T. (2007). Ionic liquids as designer solvents for nucleophilic aromatic substitutions. Org. Lett., 9(25):5247-5250.

Timofeeva, L, Kleshcheva, N. (2011). Antimicrobial polymers: mechanism of action, factors of activity, and applications. Appl. Microbial. Biotechnol., 89(3):475-492.

Wang, XS, Tabor, MW. (1988). Studies of the reactivity of morpholine, 2-mercaptobenzothiazole and 2 of their derivatives with selected amino acids. Contact Dermatitis., 19(1):16-21.

How to cite this article: Sunanda Paul, Ajoy Kumer, Md. Nuruzzaman Sarker, Mohammad Jahidul Islam, The Effect of Halogen Atoms at Propanoate Anion on Thermo Physical, Vibrational Spectroscopy, Chemical Reactivity, Biological Properties of Morpholinium Propionate Ionic Liquid. International Journal of Advanced Biological and Biomedical Research, 2020, 8(2), 112-127. Link: http://www.ijabbr.com/article 36498.html 